\title{
Efficacy analysis of trastuzumab, carboplatin and docetaxel in HER-2-positive breast cancer patients
}

\author{
DI WU and LIANGFA XIONG
}

\begin{abstract}
Department of Breast and Thyroid Surgery, The First People's Hospital of Yunnan Province, Kunming, Yunnan 650032, P.R. China
\end{abstract}

Received September 25, 2019; Accepted November 21, 2019

DOI: $10.3892 / \mathrm{ol} .2020 .11277$

\begin{abstract}
Efficacy of trastuzumab, carboplatin and docetaxel in human epidermal growth factor receptor-2 (HER-2)-positive breast cancer patients was investigated. A total of 180 HER-2-positive breast cancer patients admitted to The First People's Hospital of Yunnan Province were selected, of which 80 patients were treated with carboplatin and docetaxel and served as the control group (CG), and 100 patients were treated with trastuzumab, carboplatin and docetaxel and served as the research group (RG). Clinical efficacy, pathological efficacy, adverse reactions, inflammatory factors interleukin-6 (IL-6) and tumor necrosis factor- $\alpha$ (TNF- $\alpha$ ), cellular immune indexes of T-lymphocyte subsets $\left(\mathrm{CD}^{+}, \mathrm{CD}^{+}, \mathrm{CD}^{+} / \mathrm{CD}^{+}\right)$, and the oxidative stress indexes superoxide dismutase (SOD) and myeloperoxidase (MPO) were observed before and after treatment and were compared between both groups. Patients were followed up for 5 years, and the 5-year disease-free survival (DFS), as well as the overall survival (OS) were compared. Clinical efficacy and pathological efficacy in the RG were significantly higher than those in the CG, and the incidence rate of adverse reactions had no significant difference between the two groups. There was no significant difference in inflammatory factors, cellular immune indexes and oxidative stress indexes between the two groups before treatment. After treatment, the levels of IL-6, TNF- $\alpha, \mathrm{CD} 8^{+}$ and MPO in both groups were significantly reduced and were significantly lower in RG than those in CG. However, the levels of $\mathrm{CD}^{+}, \mathrm{CD}^{+} / \mathrm{CD}^{+}$and SOD in both groups were significantly increased after treatment and were significantly higher in RG than those in CG. The 5-year DFS and OS of the RG were significantly higher than those of the CG. In conclusion, trastuzumab, carboplatin and docetaxel present high efficacy, safety, and 5-year DFS and OS in HER-2-positive breast
\end{abstract}

Correspondence to: Dr Liangfa Xiong, Department of Breast and Thyroid Surgery, The First People's Hospital of Yunnan Province, 157 Jinbi Road, Kunming, Yunnan 650032, P.R. China

E-mail: xk28mh@163.com

Key words: trastuzumab, carboplatin, docetaxel, HER-2-positive breast cancer cancer patients, and have good recovery effect on inflammation, immune response and oxidative stress.

\section{Introduction}

Breast cancer, a high-morbidity and mortality type of cancer, is one of the leading causes of cancer-associated deaths among women worldwide $(1,2)$. According to the statistics of the American Cancer Society, 252,710 new invasive breast cancer patients and 40,610 diseased patients were estimated in 2017 (3). Human epidermal growth factor receptor-2 (HER-2) is a member of HER protein family, which plays an important role in the pathogenesis of breast cancer, regulates cyclin $\mathrm{E}$ and is related to poor viability $(4,5)$. HER-2-positive cancer is one of the most aggressive subtypes of breast cancer, which is often associated with metastasis, poor prognosis and short survival time $(6,7)$. At present, the treatment strategies for HER-2-positive breast cancer patients include local surgery, radiotherapy, systemic chemotherapy, biotherapy, and endocrine therapy, as well as the primary systemic therapy (also known as HER-2-targeted therapy and neoadjuvant therapy) which has the highest breast-conserving surgery rate $(8,9)$. In the present study, a multi-angle efficacy evaluation around a primary systemic therapy was carried out, in order to provide clinical basic data for the breast cancer treatment.

Tratuzumab is a monoclonal antibody used to target HER-2 and inhibit its function. Tratuzumab can be used in early and metastatic HER-2-positive breast cancer patients $(10,11)$. Studies have shown that trastuzumab not only has the function of cutting off HER-2 signal transmission, but can also change the immune microenvironment of tumors $(10,11)$. The genomic characteristics of tratuzumab can predict the survival of HER-2-positive breast cancer patients (12). Carboplatin is a chemotherapeutic agent widely used in malignant tumors, including breast cancer, which can be used to treat various solid malignant tumors $(13,14)$. Denkert et al $(15)$ have reported that carboplatin can enhance the interaction of chemotherapy with host immune response, having certain clinical benefits for HER-2-positive early breast cancer patients. Docetaxel is a taxane with antitumor activity, which can induce cell cycle to stagnate at $\mathrm{G} 2 / \mathrm{M}$, produce cytotoxicity and cause apoptosis $(16,17)$. At present, docetaxel is considered one of the drugs used in the first-line combination therapy of HER-2-positive metastatic breast cancer patients (18). The 
aforementioned three drugs have been applied to the treatment of HER-2-positive early and locally advanced breast cancer patients as a therapeutic scheme with good tolerance, high safety and no long-term toxicity or side-effects (19).

Currently, there are few researches on the efficacy evaluation of the combined treatment of the three drugs for HER-2-breast cancer patients. In the present study, the efficacy, safety, survival and changes of relevant indicators of these drugs were observed and analyzed.

\section{Patients and methods}

General information. A total of 180 HER-2-positive breast cancer female patients, admitted to The First People's Hospital of Yunnan Province (Kunming, China) from January 2013 to June 2014, were enrolled in this study. Eighty patients were selected as the control group (CG) and were treated with carboplatin and docetaxel, and 100 patients were selected as the research group (RG) and were treated with trastuzumab carboplatin and docetaxel. The patients in the CG were 22-78 years of age with an average age of $48.9 \pm 5.7$ years, and the patients in the RG were 25-79 years of age with an average age of $50.1 \pm 5.9$ years. The study was approved by the Ethics Committee of The First People's Hospital of Yunnan Province. Signed informed consents were obtained from the patients and/or guardians.

Inclusion and exclusion criteria. The inclusion criteria were as follows: Patients with HER-2-positive breast cancer, as diagnosed by a histopathological examination (20); patients who had not received any treatment method; patients with no history of surgery; patients that accepted a 5-year follow-up and were willing to cooperate for this research; and patients who had complete clinicopathological data. The exclusion criteria were as follows: Patients with contraindications or allergic history to the treatment; patients with breast tissue inflammation; patients with hyperthyroidism and other diseases that could affect the results of the study; patients with other serious organ dysfunction; patients with malignant tumors in the past; pregnant women. Inclusion criteria were applicable to all subjects.

Treatment methods. Patients in both groups were treated with conventional therapy. The patients in CG received intravenous drip of carboplatin with $\mathrm{AUC}=5 \mathrm{mg} / \mathrm{ml} / \mathrm{min}$ and docetaxel with $\mathrm{AUC}=75 \mathrm{mg} / \mathrm{m}^{2}$ (J55611 and $\mathrm{J} 43650$, respectively; Shanghai Jinsui Biotechnology Co., Ltd.; ) once every 3 weeks for 4-6 cycles of treatment. The patients in RG received intravenous drip of carboplatin, docetaxel and trastuzumab (TM-Tras-00002_1; Shanghai TheraMabs Biotechnology Co., Ltd.), $8 \mathrm{mg} / \mathrm{kg}$ for the first time and $6 \mathrm{mg} / \mathrm{kg}$ for the second time, once every 3 weeks, for 17 cycles of treatment.

Efficacy evaluation. Clinical efficacy was evaluated according to the efficacy evaluation criteria of solid tumors (RECIST version 1.1) (21): Complete remission (CR) was considered when the lesion completely disappeared and the duration was $\geq 4$ weeks. Partial remission (PR) was considered when the reduction of the lesion's longest diameter was $\geq 30 \%$ and the duration was $\geq 4$ weeks. The occurrence of new lesions and increase of lesion length $\geq 20 \%$ was considered as progressive disease (PD). Stable disease (SD) was considered when the long diameter of lesions decreased or increased, and the disease could be characterized between PR and PD. According to the efficacy of both groups, CR and PR were defined as effective and the total effective rate was calculated as $(\mathrm{CR}+\mathrm{PR}) /($ total no. of cases) $\times 100 \%$.

Pathological efficacy was evaluated with reference to the Miller-Payne grading system (22) and was defined according to the lesion density or the reduction percentage of the reaction volume: $\mathrm{G}_{1}$ was $0 \%, \mathrm{G}_{2}$ was $<33 \%, \mathrm{G}_{3}$ was $33-66 \%, \mathrm{G}_{4}$ was $67-99 \%$ and $G_{5}$ was $100 \% . G_{1}$ to $G_{5}$ describe the condition of tumor cells in lesions from no improvement to necrosis or disappearance. According to the efficacy of both groups, $\mathrm{G}_{3}$, $\mathrm{G}_{4}$ and $\mathrm{G}_{5}$ were defined as effective and the total effective rate was calculated as $\left(\mathrm{G}_{3}+\mathrm{G}_{4}+\mathrm{G}_{5}\right) /($ total no. of cases) $\mathrm{x} 100 \%$.

Observational indicators. Clinical efficacy, pathological efficacy, adverse reactions, inflammatory factors interleukin-6 (IL-6) and tumor necrosis factor- $\alpha$ (TNF- $\alpha$ ), cellular immune indexes T-lymphocyte subsets $\left(\mathrm{CD} 4^{+}, \mathrm{CD}^{+}, \mathrm{CD} 4^{+} / \mathrm{CD}^{+}\right)$, and the oxidative stress indexes superoxide dismutase (SOD) and myeloperoxidase (MPO) before and after treatment, as well as the 5-year disease-free survival (DFS) and overall survival (OS) of patients, were observed and compared between the two groups.

Detection methods. Before and after treatment, $3 \mathrm{ml}$ of elbow venous blood were collected from the patients of both groups, and placed into anticoagulant-free and EDTA-K2 blood collection vessels. The peripheral blood T-lymphocyte subsets were detected by flow cytometry. A total of $10 \mu 1$ of fluorescein isothiocyanate (FITC)-labeled fluorescent monoclonal antibody (CD4-FITC/CD8-ECD) (1:200; RM25013 and 737659, respectively; China Shanghai Haoran Biotechnology Co., Ltd.) were added to each test tube, and $100 \mu \mathrm{l}$ of venous blood from the EDTA-K2 blood collection vessel were added and mixed evenly. The samples were left in the dark at room temperature for $20 \mathrm{~min}$. Next, $500 \mu 1$ of red blood cell lysis buffer were added to lyse erythrocytes, and the samples were left in the dark at room temperature for $15 \mathrm{~min}$; $500 \mu \mathrm{l}$ of PBS buffer were added and mixed well, and the mixture was left at room temperature for $10 \mathrm{~min}$ in the dark. Samples were detected by a flow cytometer (NovoCyte ${ }^{\mathrm{TM}}$; ACEA Biosciences, Inc.), and the $\mathrm{CD}^{+}, \mathrm{CD}^{+}$and $\mathrm{CD} 4^{+} / \mathrm{CD}^{+}$data were analyzed using CellQuest software (Becton, Dickinson and Co.).

The venous blood in the anticoagulant-free blood collection vessel was placed on a centrifuge and was centrifuged at $1,500 \mathrm{x} \mathrm{g}$ at $4^{\circ} \mathrm{C}$ for $10 \mathrm{~min}$. The separated upper serum was stored in a refrigerator at $-20^{\circ} \mathrm{C}$ for later use. The concentrations of serum IL-6, TNF- $\alpha$, SOD and MPO were detected by ELISA (23), according to the manufacturer's instructions of IL-6, TNF- $\alpha$, SOD and MPO detection kits (Shanghai Fanke Biotechnology Co., Ltd.). Blank wells (without any reagent), standard wells and sample wells to be tested were set up. A total of 50 liters of standard substance were added to the standard substance well, 50 liters of sample were added to the sample wells, and 50 liters of streptavidin-HRP were added to each well. The plate was sealed and the temperature 
Table I. Comparison of clinicopathological characteristics of patients in the two groups [n (\%), mean \pm SD].

\begin{tabular}{|c|c|c|c|c|}
\hline Clinicopathological characteristics & Control group $(n=80)$ & Research group $(n=100)$ & $\chi^{2} / \mathrm{t}$ value & P-value \\
\hline Age (years) & & & 0.180 & 0.671 \\
\hline$<35$ & $8(10.00)$ & $12(12.00)$ & & \\
\hline$\geq 35$ & $72(90.00)$ & $88(88.00)$ & & \\
\hline Menstrual status & & & 0.422 & 0.650 \\
\hline Before menopause & $29(36.25)$ & $41(41.00)$ & & \\
\hline After menopause & $51(63.75)$ & $59(59.00)$ & & \\
\hline Histological classification & & & 0.321 & 0.852 \\
\hline $\mathrm{I}$ & $2(2.50)$ & $4(4.00)$ & & \\
\hline II & $23(28.75)$ & $29(29.00)$ & & \\
\hline III & $55(68.75)$ & $67(67.00)$ & & \\
\hline Hormone receptor status & & & 1.608 & 0.205 \\
\hline Positive & $34(42.50)$ & $52(52.00)$ & & \\
\hline Negative & $46(57.50)$ & $48(48.00)$ & & \\
\hline T staging & & & 0.302 & 0.583 \\
\hline $1 / 2$ & $71(88.75)$ & $86(86.00)$ & & \\
\hline $3 / 4$ & $9(11.25)$ & $14(14.00)$ & & \\
\hline $\mathrm{N}$ staging & & & 0.384 & 0.825 \\
\hline 0 & $35(43.75)$ & $41(41.00)$ & & \\
\hline 1 & $34(42.50)$ & $42(42.00)$ & & \\
\hline 2 & $11(13.75)$ & $17(17.00)$ & & \\
\hline TNM staging & & & 1.381 & 0.501 \\
\hline I & $2(2.50)$ & $5(5.00)$ & & \\
\hline II & $60(75.00)$ & $68(68.00)$ & & \\
\hline III & $18(22.50)$ & $27(27.00)$ & & \\
\hline Breast surgery & & & 0.497 & 0.481 \\
\hline Breast-conserving & $7(8.75)$ & $12(12.00)$ & & \\
\hline Total removal & $73(91.25)$ & $88(88.00)$ & & \\
\hline Adjuvant endocrine therapy & & & 0.360 & 0.549 \\
\hline Yes & $42(52.50)$ & $48(48.00)$ & & \\
\hline No & $38(47.50)$ & $52(52.00)$ & & \\
\hline Postoperative radiotherapy & & & 0.120 & 0.729 \\
\hline Yes & $50(62.50)$ & $65(65.00)$ & & \\
\hline No & $30(37.50)$ & $35(35.00)$ & & \\
\hline Tumor diameter $(\mathrm{cm})$ & $4.37 \pm 1.35$ & $4.49 \pm 1.47$ & 0.564 & 0.573 \\
\hline Place of residence & & & 0.587 & 0.444 \\
\hline Countryside & $26(32.50)$ & $38(38.00)$ & & \\
\hline Cities and towns & $54(67.50)$ & $62(62.00)$ & & \\
\hline
\end{tabular}

was kept at $37^{\circ} \mathrm{C}$ for 60 min. Liquid was discarded, and the plate was washed by washing liquid, and then spin-dried. This procedure was repeated 5 times. Developer A (50 liters) and developer B (50 liters) were added to each well and mixed well, and the wells were placed stably in the dark at $37^{\circ} \mathrm{C}$ for $10 \mathrm{~min}$. Next, $50 \mu \mathrm{l}$ of stop solution were added to each well. BioTek full-automatic microplate reader (800TS; Shanghai BioExcellence Co., Ltd.) was used. The blank wells were zeroed and the absorbance value (OD value) of each well was sequentially measured at $450 \mathrm{~nm}$ wavelength. The concentrations of IL-6, TNF- $\alpha$, SOD, and MPO were calculated.
Follow-up. Patients were followed up 4 times/year for 5 years through telephone calls, visits and consulting pathological data. DFS time was defined as the period from the first treatment of the patient till the first relapse of the disease, and OS time was defined as the period from the diagnosis of the disease till the death of the patient or the last follow-up day.

Statistical analysis. GraphPad Prism 6 software (GraphPad Software, Inc.) was used for the statistical analysis of the data, and the production and analysis of the figures. Counting data were expressed by the number of cases and percentage [n (\%)], 
Table II. Comparison of clinical efficacy between the two groups before and after treatment [n (\%)].

\begin{tabular}{lcccccc}
\hline Group & $\mathrm{n}$ & $\mathrm{CR}$ & $\mathrm{PR}$ & $\mathrm{SD}$ & $\mathrm{PD}$ & Total efficacy \\
\hline CG & 80 & $15(18.75)$ & $27(33.75)$ & $20(25.00)$ & $18(22.50)$ & $52.50 \%$ \\
RG & 100 & $18(18.00)$ & $58(58.00)$ & $18(18.00)$ & $6(6.00)$ & $76.00 \%$ \\
$\chi^{2}$ value & - & - & - & - & - & - \\
P-value & - & - & - & - & - & 0.001 \\
\hline
\end{tabular}

CR, complete remission; PR, partial remission; SD, stable disease; PD, progressive disease; CG, control group; RG, research group.

Table III. Comparison of pathological efficacy between the two groups before and after treatment [n (\%)].

\begin{tabular}{lccccccc}
\hline Group & $\mathrm{n}$ & $\mathrm{G}_{1}$ & $\mathrm{G}_{2}$ & $\mathrm{G}_{3}$ & $\mathrm{G}_{4}$ & $\mathrm{G}_{5}$ & Total efficacy \\
\hline CG & 80 & $20(25.00)$ & $28(35.00)$ & $18(22.50)$ & $12(15.00)$ & $2(2.50)$ & $40.00 \%$ \\
RG & 100 & $13(13.00)$ & $24(24.00)$ & $33(33.00)$ & $22(22.00)$ & $8(8.00)$ & $63.00 \%$ \\
$\chi^{2}$ value & - & - & - & - & - & - & 9.434 \\
P-value & - & - & - & - & - & - & 0.002 \\
\hline
\end{tabular}

$\mathrm{CG}$, control group; RG, research group.

Table IV. Comparison of the adverse reactions in the two groups [n (\%)].

\begin{tabular}{lcccr}
\hline Category & CG $(\mathrm{n}=80)$ & RG $(\mathrm{n}=100)$ & $\chi^{2}$ value & P-value \\
\hline III-IV degree myelosuppression & $20(25.00)$ & $28(28.00)$ & 0.205 & 0.651 \\
III-IV degree gastrointestinal reaction & $9(11.25)$ & $13(13.00)$ & 0.127 & 0.722 \\
Liver function damage & $6(7.50)$ & $8(8.00)$ & 0.010 & 0.920 \\
Cardiac toxicity & $0(0.00)$ & $1(1.00)$ & 0.804 & 0.370 \\
Peripheral neurotoxicity & $9(11.25)$ & $12(12.00)$ & 0.024 & 0.876 \\
\hline
\end{tabular}

CG, control group; RG, research group.

and Chi-square test was used for their comparisons between groups. Measurement data were expressed as the mean \pm SD and their comparison between two groups was conducted by the independent samples t-test, whereas for the comparisons between multiple groups ANOVA with LSD post hoc test were carried out. Kaplan-Meier method was used to analyze the DFS and OS of the patients, and log-rank test was used to evaluate the differences in survival time between the two groups. $\mathrm{P}<0.05$ was considered to indicate a statistically significant difference.

\section{Results}

Clinicopathological data. There was no significant difference between the two groups in age, menstrual status, histological grade, hormone receptor status, T staging, N staging, TNM staging, breast surgery, adjuvant endocrine therapy, postoperative radiotherapy, tumor diameter, or place of residence $(\mathrm{P}>0.05)$ (Table I).

Comparison of clinical efficacy. Clinical efficacy results for the CG showed that CR, PR, SD and PD cases were 15, 27,
20 and 18 , respectively, with a total effective rate of $52.50 \%$. The clinical efficacy results for the RG showed that CR, PR, SD and PD cases were 18, 58, 18 and 6, respectively, with a total effective rate of $76.00 \%$. The total effective rate in the $R G$ was significantly higher than that of the $C G$ $(\mathrm{P}<0.001)$ (Table II).

Comparison of pathological efficacy. Pathological efficacy results for the $C G$ showed that $G_{1}, G_{2}, G_{3}, G_{4}$ and $G_{5}$ cases were $20,28,18,12$ and 2 , respectively, with a total effective rate of $40.00 \%$. The pathological efficacy results for the RG showed that $G_{1}, G_{2}, G_{3}, G_{4}$ and $G_{5}$ cases were $13,24,33,22$ and 8 , respectively, with a total effective rate of $63.00 \%$. The total effective rate in the RG was significantly higher than that of the $\mathrm{CG}(\mathrm{P}<0.01)$ (Table III).

Comparison of adverse reactions. After treatment, the adverse reactions in both groups of patients included of III-IV degree myelosuppression, III-IV degree gastrointestinal reaction, liver function damage, cardiac toxicity, and peripheral neurotoxicity. The main adverse reactions were III-IV degree myelosuppression, III-IV degree gastrointestinal reaction, and 

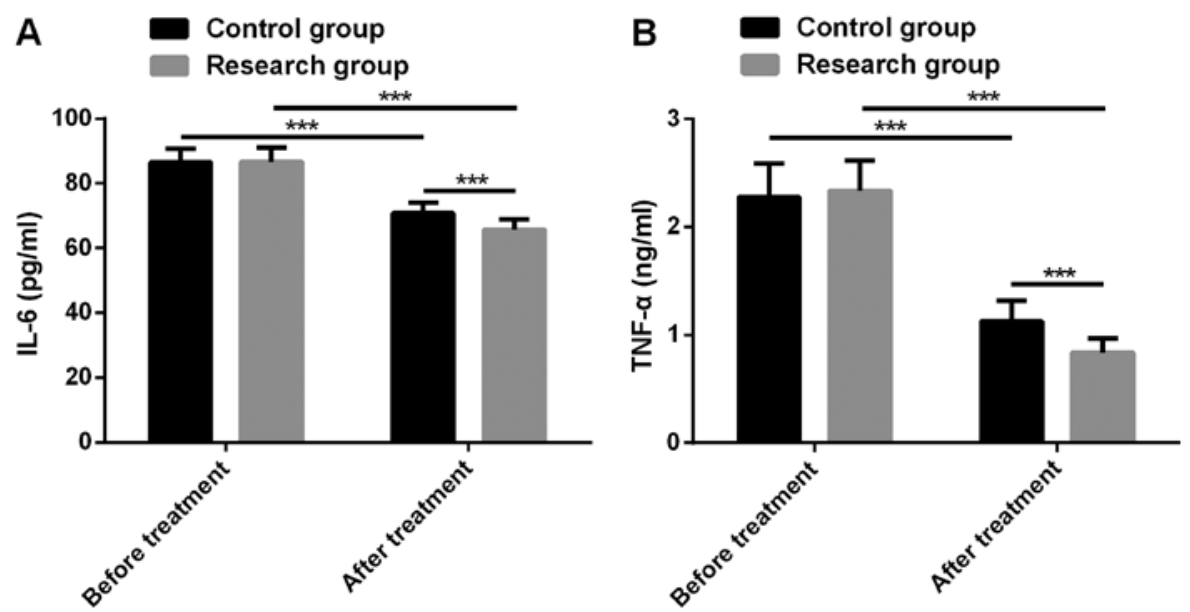

Figure 1. Changes of the levels of inflammatory factors in both groups before and after treatment. Inflammatory factor (A) IL-6 and (B) TNF- $\alpha$ decreased significantly in both groups after treatment. ${ }^{* * *} \mathrm{P}<0.001$. IL-6, interleukin-6; TNF- $\alpha$, tumor necrosis factor- $\alpha$.


Figure 2. Changes of the cellular immune indexes in both groups before and after treatment. (A) Cellular immune index CD4+ increased significantly in both groups after treatment. (B) Cellular immune index $\mathrm{CD}^{+}$decreased significantly in both groups after treatment. (C) Cellular immune indexes $\mathrm{CD}^{+} / \mathrm{CD}^{+}$ increased significantly in both groups after treatment. ${ }^{* * *} \mathrm{P}<0.01$ and ${ }^{* * *} \mathrm{P}<0.001$.

peripheral neurotoxicity. The incidence rate of adverse reactions in both groups of patients had no significant difference $(\mathrm{P}>0.05)$ (Table IV).

Changes of the levels of inflammatory factors. There was no significant difference in the levels of inflammatory factors between RG and CG before treatment $(\mathrm{P}>0.05)$. After treatment, IL-6 and TNF- $\alpha$ levels decreased significantly in both groups $(\mathrm{P}<0.001)$, and the levels in $\mathrm{RG}$ were significantly lower than those in $\mathrm{CG}(\mathrm{P}<0.001)$ (Fig. 1).

Changes of cellular immune indexes. There was no significant difference in cellular immune indexes between RG and CG before treatment $(\mathrm{P}>0.05)$. After treatment, $\mathrm{CD} 4^{+}, \mathrm{CD} 4^{+} / \mathrm{CD}^{+}$ levels increased significantly in both groups $(\mathrm{P}<0.001)$, and the levels in RG were significantly higher than those in CG $(\mathrm{P}<0.001)$. However, $\mathrm{CD} 8^{+}$decreased significantly in both groups after treatment $(\mathrm{P}<0.001)$, and $\mathrm{CD}^{+}$level was significantly lower in $\mathrm{RG}$ than that in $\mathrm{CG}(\mathrm{P}<0.01)$ (Fig. 2).

Changes of oxidative stress indexes. There was no significant difference in oxidative stress indexes between RG and CG before treatment $(\mathrm{P}>0.05)$. After treatment, $\mathrm{SOD}$ of patients in both groups increased significantly $(\mathrm{P}<0.001)$, and SOD level in RG was significantly higher than that in CG $(\mathrm{P}<0.01)$. However, MPO in both groups after treatment reduced significantly $(\mathrm{P}<0.001)$, and MPO level in RG was significantly lower than that in $\mathrm{CG}(\mathrm{P}<0.001)$ (Fig. 3).

Survival analysis. A total of 180 patients were successfully followed up for 5 years. The 5-year DFS and OS of RG were significantly higher than those of $\mathrm{CG}(\mathrm{P}<0.05)$ (Fig. 4).

\section{Discussion}

Inflammatory response in tumor microenvironment is related to poor prognosis in breast cancer, which may be related to the involvement of inflammatory mediators in stimulating proliferation, invasion and angiogenesis of breast cancer cells (24). Thriveni et al (25) have reported that TNF- $\alpha$ is expressed in the plasma of patients with invasive cancer diseases at high levels, as an inflammatory microenvironment marker for patients with primary breast cancer. IL-6 is an inflammatory medium related to the activity of cancer cells. The amplification or 

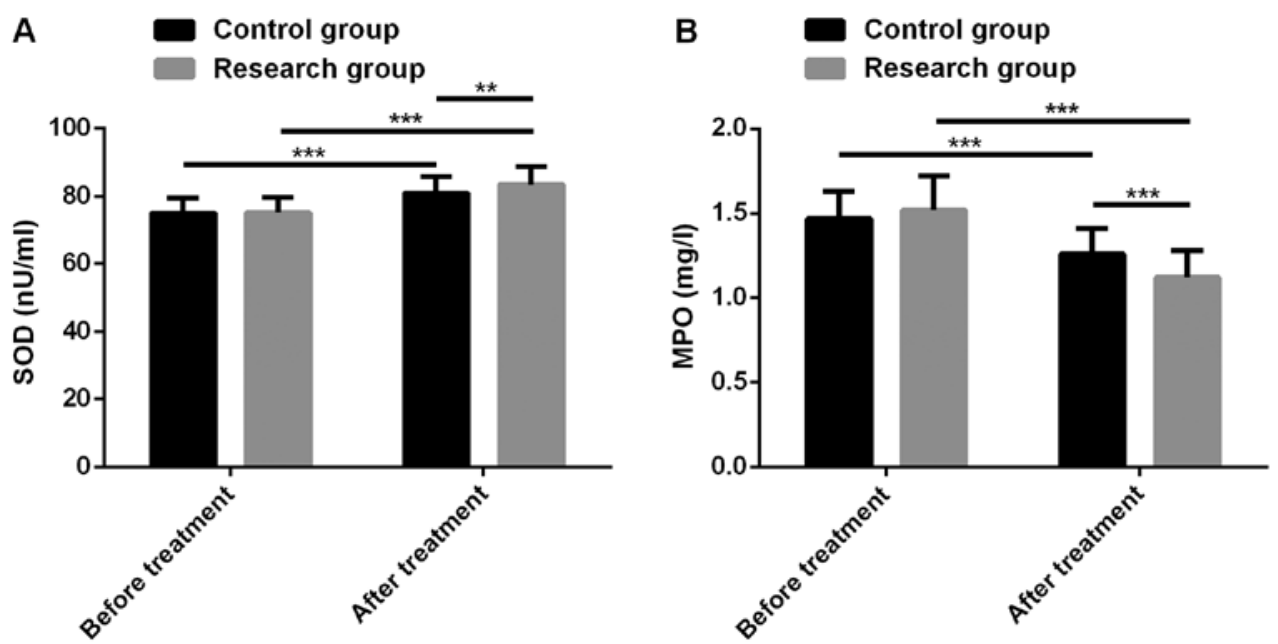

Figure 3. Changes of the oxidative stress indexes in both groups before and after treatment. (A) Oxidative stress index SOD increased significantly in both groups after treatment. (B) Oxidative stress index MPO decreased significantly in both groups after treatment. ${ }^{* *} \mathrm{P}<0.01$ and ${ }^{* * *} \mathrm{P}<0.001$. SOD, superoxide dismutase; MPO, myeloperoxidase.
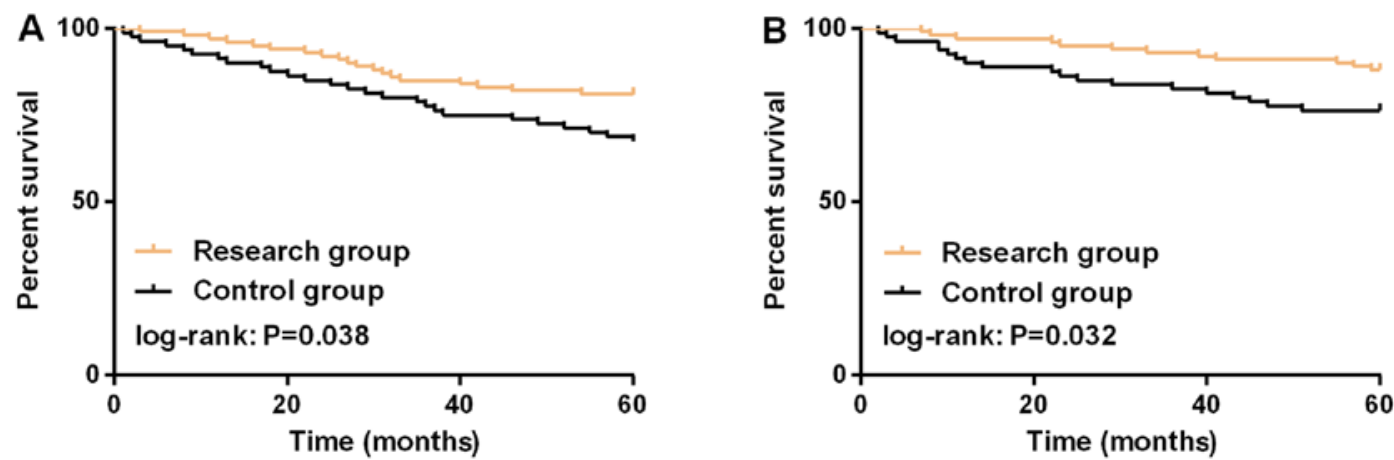

Figure 4. Comparison of survival analysis results. The 5-year (A) disease-free survival and (B) overall survival of the patients in the RG were significantly higher than those in the CG. RG, research group; CG, control group.

overexpression of IL-6 has a certain predictive ability for the recurrence of estrogen or progesterone receptor-positive breast cancer in females $(26,27)$. Co-expression of serum IL-6 and TNF- $\alpha$ can be used as an effective tumor marker for tumor invasion and breast cancer prognosis (28). In the present study, the levels of inflammatory factors IL- 6 and TNF- $\alpha$ of patients in both groups were significantly reduced after treatment, and the levels in the RG were significantly lower than those in CG, indicating that trastuzumab, carboplatin and docetaxel have better recovery effect for patients with inflammatory imbalance. Seo et al (29) have considered that the infiltration of T-lymphocyte subsets is tied to the phenotype of breast cancer stem cells and epithelial-mesenchymal transformation. T-lymphocyte subsets, as indicators of immune function, have a certain predictive ability for tumor progression and lymph node metastasis. $\mathrm{CD} 4$ and $\mathrm{CD} 4^{+} / \mathrm{CD}^{+}$have been reported to be negatively correlated with breast cancer tumor progression whereas, a positive correlation has been reported for $\mathrm{CD}^{+}(30)$. In the present study, $\mathrm{CD} 4^{+}$and $\mathrm{CD} 4^{+} / \mathrm{CD} 8^{+}$had higher expression levels in $\mathrm{RG}$ after treatment, while $\mathrm{CD} 8^{+}$ expression level was lower in RG after treatment, indicating that trastuzumab, carboplatin and docetaxel have better effect on relieving immunosuppression in patients. Oxidative stress balance is involved in the occurrence and development of breast cancer. Oxidation and anti-oxidant preparations play an important part in the regulation of breast cancer. Low level SOD has been linked to the occurrence and development of breast cancer (31). Recently, studies have also reported that SOD mimetics, which mimic SOD performance, have inhibitory effects on the migration, invasion and angiogenesis of breast cancer cells and can be used as drugs in redox therapy for breast cancer (32). MPO is an endogenous metabolic/oxidative lysosomal enzyme secreted by neutrophils and monocytes, which can play a crucial role in tumor invasion by activating carcinogens into genotoxic intermediates and then enhancing xenogenic carcinogenicity (33). As reported, breast cancer patients have higher MPO levels, which may also reflect the oxidative stress imbalance of the disease (34). As to the role of oxidative stress in breast cancer, Zapf et al (35) have stated that breast cancer patients have low levels of SOD and high levels of MPO, and simple chemotherapy would aggravate the oxidative stress levels of both. In the present study, the patients of the RG had higher level of SOD and lower level of MPO after treatment, suggesting that trastuzumab, carboplatin and docetaxel have more gratifying effects on reversing the oxidative stress imbalance of the patients. 
The results of the study revealed that the clinical efficacy and pathological efficacy in the RG were significantly higher than those in the $\mathrm{CG}$, and there was no significant difference in the incidence rate of adverse reactions between the two groups. Finally, a 5-year follow-up of the patients in both groups was conducted. Compared with the patients in the $\mathrm{CG}$, the patients in the RG had higher 5-year DFS and OS, indicating that trastuzumab, carboplatin and docetaxel could significantly improve the 5-year DFS and OS of patients.

The present study also confirmed the clinical benefits of trastuzumab, carboplatin and docetaxel; however, there is still room for improvement. First of all, further research could be conducted on cell biology and the specific regulatory mechanism on breast cancer cells. Secondly, a larger sample size should be included in order to improve the accuracy of the research results.

In conclusion, trastuzumab, carboplatin and docetaxel can be potentially used in clinic for the HER-2 positive breast cancer treatment.

\section{Acknowledgements}

Not applicable.

\section{Funding}

No funding was received.

\section{Availability of data and materials}

The datasets used and/or analyzed during the present study are available from the corresponding author on reasonable request.

\section{Authors' contributions}

DW and LX conceived and designed the study. DW and LX were responsible for the collection, analysis and interpretation of the data. DW drafted the manuscript. LX revised the manuscript critically for important intellectual content. All authors read and approved the final manuscript.

\section{Ethics approval and consent to participate}

The study was approved by the Ethics Committee of The First People's Hospital of Yunnan Province (Kunming, China). Signed informed consents were obtained from the patients and/or guardians.

\section{Patient consent for publication}

Not applicable.

\section{Competing interests}

The authors declare that they have no competing interests.

\section{References}

1. Asif HM, Sultana S, Ahmed S, Akhtar N and Tariq M: HER-2 positive breast cancer-a mini-review. Asian Pac J Cancer Prev 17: $1609-1615,2016$
2. Ghislain I, Zikos E, Coens C, Quinten C, Balta V, Tryfonidis K, Piccart M, Zardavas D, Nagele E, Bjelic-Radisic V, et al; European Organisation for Research and Treatment of Cancer (EORTC) Quality of Life Group; Breast Cancer Group; EORTC Headquarters: Health-related quality of life in locally advanced and metastatic breast cancer: Methodological and clinical issues in randomised controlled trials. Lancet Oncol 17: e294-e304, 2016.

3. DeSantis CE, Ma J, Goding Sauer A, Newman LA and Jemal A: Breast cancer statistics, 2017, racial disparity in mortality by state. CA Cancer J Clin 67: 439-448, 2017.

4. Suzawa K, Toyooka S, Sakaguchi M, Morita M, Yamamoto H, Tomida S, Ohtsuka T, Watanabe M, Hashida S, Maki Y, et al: Antitumor effect of afatinib, as a human epidermal growth factor receptor 2-targeted therapy, in lung cancers harboring HER2 oncogene alterations. Cancer Sci 107: 45-52, 2016.

5. Luhtala S, Staff S, Tanner M and Isola J: Cyclin E amplification, over-expression, and relapse-free survival in HER-2-positive primary breast cancer. Tumour Biol 37: 9813-9823, 2016.

6. Grosset AA, Poirier F, Gaboury L and St-Pierre Y: Galectin-7 expression potentiates her-2-positive phenotype in breast cancer. PLoS One 11: e0166731, 2016.

7. Yang F, Lyu S, Dong S, Liu Y, Zhang X and Wang O: Expression profile analysis of long noncoding RNA in HER-2-enriched subtype breast cancer by next-generation sequencing and bioinformatics. OncoTargets Ther 9: 761-772, 2016.

8. Gradishar WJ, Anderson BO, Balassanian R, Blair SL, Burstein HJ, Cyr A, Elias AD, Farrar WB, Forero A, Giordano SH, et al: Breast cancer version 2.2015. J Natl Compr Canc Netw 13: 448-475, 2015.

9. Ahmed S, Sami A and Xiang J: HER2-directed therapy: Current treatment options for HER2-positive breast cancer. Breast Cancer 22: 101-116, 2015.

10. Shi SJ, Wang LJ, Yu B, Li YH, Jin Y and Bai XZ: LncRNA-ATB promotes trastuzumab resistance and invasion-metastasis cascade in breast cancer. Oncotarget 6: 11652-11663, 2015.

11. Perez EA, Barrios C, Eiermann W, Toi M, Im YH, Conte P, Martin M,Pienkowski T, Pivot X, Burris H III, et al: Trastuzumab emtansine with or without pertuzumab versus trastuzumab plus taxane for human epidermal growth factor receptor 2-positive, advanced breast cancer: Primary results from the phase III MARIANNE study. J Clin Oncol 35: 141-148, 2017.

12. Hendricks WPD, Briones N, Halperin RF, Facista S, Heaton PR, Mahadevan D and Kim S: Genomic signature of trastuzumab neoadjuvant therapy predictive of patient survival in HER2-positive breast cancer. Cancers (Basel) 11: E1566, 2019.

13. Ho GY, Woodward N and Coward JI: Cisplatin versus carboplatin: Comparative review of therapeutic management in solid malignancies. Crit Rev Oncol Hematol 102: 37-46, 2016.

14. Theile D: Under-reported aspects of platinum drug pharmacology. Molecules 22: 382, 2017.

15. Denkert C, Loibl S, Salat C, Sinn BV, Schem C, Endris V, Klare P, Schmitt WD, Blohmer JU, Weichert W, et al: Abstract S1-06: Increased tumor-associated lymphocytes predict benefit from addition of carboplatin to neoadjuvant therapy for triple-negative and HER2-positive early breast cancer in the GeparSixto trial (GBG 66). Cancer Res 73 (abstract nr S1-06), 2013.

16. Clarke SJ and Rivory LP: Clinical pharmacokinetics of docetaxel. Clin Pharmacokinet 36: 99-114, 1999.

17. Lyseng-Williamson KA and Fenton C: Docetaxel: A review of its use in metastatic breast cancer. Drugs 65: 2513-2531, 2005.

18. Leung HW, Chan AL, Muo CH and Leung JH: Cost-effectiveness of pertuzumab combined with trastuzumab and docetaxel as a first-line treatment for HER-2 positive metastatic breast cancer. Expert Rev Pharmacoecon Outcomes Res 18: 207-213, 2018.

19. Echavarria I, Granja M, Bueno C, Lopez-Tarruella S, Peinado P, Sotelo M, Jerez Y, Moreno F, Torres G, Lobo M, et al: Multicenter analysis of neoadjuvant docetaxel, carboplatin, and trastuzumab in HER2-positive breast cancer. Breast Cancer Res Treat 162: 181-189, 2017.

20. Kataja V and Castiglione M; ESMO Guidelines Working Group: Primary breast cancer: ESMO clinical recommendations for diagnosis, treatment and follow-up. Ann Oncol 20 (Suppl 4): 10-14, 2009.

21. Keil S, Barabasch A, Dirrichs T, Bruners P, Hansen NL, Bieling HB, Brümmendorf TH and Kuhl CK: Target lesion selection: An important factor causing variability of response classification in the Response Evaluation Criteria for Solid Tumors 1.1. Invest Radiol 49: 509-517, 2014.

22. Shintia C, Endang H and Diani K: Assessment of pathological response to neoadjuvant chemotherapy in locally advanced breast cancer using the Miller-Payne system and TUNEL. Malays J Pathol 38: 25-32, 2016. 
23. Hornbeck PV: Enzyme-linked immunosorbent assays. Curr Protoc Immunol 110: 2.1.1-23, 2015.

24. Cho YA, Sung MK, Yeon JY, Ro J and Kim J: Prognostic role of interleukin-6, interleukin-8, and leptin levels according to breast cancer subtype. Cancer Res Treat 45: 210-219, 2013

25. Thriveni K, Raju A, Ramaswamy G, Shachidevi Krishnamurthy $S$ and Kumar R: Tumor necrosis factor: An inflammatory microenvironment marker in primary breast cancer patients. IJMIO 3 : 25,2018

26. Drygin D, Ho CB, Omori M, Bliesath J, Proffitt C, Rice R, Siddiqui-Jain A, O' Brien S, Padgett C, Lim JK, et al: Protein kinase CK2 modulates IL-6 expression in inflammatory breast cancer. Biochem Biophys Res Commun 415: 163-167, 2011.

27. Muss HB, Bunn JY, Crocker A, Plaut K, Koh J,Heintz N, Rincon M, Weaver DL, Tam D, Beatty B, et al: Cyclin D-1, interleukin-6, HER-2/neu, transforming growth factor receptor-II and prediction of relapse in women with early stage, hormone receptor-positive breast cancer treated with tamoxifen. Breast J 13: 337-345, 2007.

28. Tripsianis G, Papadopoulou E, Anagnostopoulos K, Botaitis S, Katotomichelakis M, Romanidis K, Kontomanolis E, Tentes I and Kortsaris A: Coexpression of IL-6 and TNF- $\alpha$ : Prognostic significance on breast cancer outcome. Neoplasma 61: 205-212, 2014.

29. Seo AN, Lee HJ, Kim EJ, Kim HJ, Jang MH, Lee HE, Kim YJ, Kim JH and Park SY: Tumour-infiltrating CD8 $8^{+}$lymphocytes as an independent predictive factor for pathological complete response to primary systemic therapy in breast cancer. Br J Cancer 109: 2705-2713, 2013.
30. Wang B and Pan F: Analysis of T lymphocyte subsets in peripheral blood of patients with breast cancer and its clinical value. Int J Lab Med 39: 1230-1232, 1237, 2018.

31. Sahu A, Varma M and Kachhawa K: A prognostic study of MDA, SOD and catalase in breast Cancer patients. Int J Sci Res (Ahmedabad) 4: 157-159, 2015.

32. Fernandes AS, Saraiva N and Oliveira NG: Redox therapeutics in breastcancer: Role of SODmimics.In:Redox-Active Therapeutics. Batinic-Haberle I, Reboucas JS and Spasojevic I (eds). Springer, Cham, Switzerland, pp451-467, 2016.

33. Yang WJ, Wang MY, Pan FZ, Shi C and Cen H: Association between MPO-463G $>$ A polymorphism and cancer risk: Evidence from 60 case-control studies. World J Surg Oncol 15: 144, 2017.

34. Abusoglu S, Eryavuz D, Bal C, Nural C, Ozcan E, Yildirimel M, Celik S and Unlu A: Assessment of serum ischemia-modified albumin, prolidase and thiol-disulphide levels in subjects with breast cancer. Rev Rom Med Lab 27: 25-33, 2019.

35. Zapf I, Moezzi M, Fekecs T, Nedvig K, Lőrinczy D and Ferencz A: Influence of oxidative injury and monitoring of blood plasma by DSC on breast cancer patients. J Therm Anal Calorim 123: 2029-2035, 2016. 